Universität Bielefeld Faculty of Business Administration and Economics

Working Papers in Economics and Management

No. 12-2019

December 2019

\title{
Endogeneous strategy spaces: Simultaneous move vs. sequential move games
}

Nikolai M. Brandt

\author{
Dennis Heitmann
}




\title{
Endogeneous strategy spaces: Simultaneous move vs. sequential move games
}

\author{
Nikolai M. Brandt* \& Dennis Heitmann ${ }^{\dagger}$
}

December 4, 2019

\begin{abstract}
In a horizontal differentiated duopoly we compare Nash and Stackelberg equilibria in which the firms endogeneously choose to behave as a price or quantity setter. Using the utility function introduced by Dixit (1979) we generalize the model of Boyer and Moreaux (1987) and show that it is always more profitable to strategically set the price (quantity) if the goods are complements (substitutes). For every degree of product differentiation, consumer surplus and total welfare are maximal in the standard Bertrand equilibrium, followed by the price Stackelberg, the quantity Stackelberg and the Cournot equilibrium. In contrast to Boyer and Moreaux we show that there is no unique ranking of prices, quantities and profits of the leader and follower depending on the degree of product differentiation and the type of competition. Furthermore, we show that the price (quantity) Stackelberg equilibrium is bounded by the Bertrand and the mixed Nash equilibrium in which firm 1 sets the price (quantity) and firm 2 the quantity (price).
\end{abstract}

JEL classification numbers: C72; D43; L13

Keywords: Stackelberg equilibrium; Cournot; Bertrand; strategy space

\section{Introduction}

The Stackelberg game is a standard model in oligopoly theory, which is one of the most intensively discussed topics in industrial economics and based on the pioneering works of Cournot (1838) and Betrand (1883). In the former, the firms

*Bielefeld University, Germany, nbrandt@ uni-bielefeld.de

${ }^{\dagger}$ BSP Campus Hamburg, Germany, dennis.heitmann @bsp-campus-hamburg.de 
simultaneously choose quantities while in the latter prices are the strategic variables. Despite these classical simultaneous move games, the model of Stackelberg (1934) describes a situation with asymmetric information in which one firm, the leader, decides first. After observing this, the follower choose its own optimal strategy. There is a huge volume of these models for homogeneous as well as for horizontal differentiated goods (see e.g. Amir and Jin (2001), Dastidar (2004), Kreps and Scheinkman (1983), Vives (1985) and Vives (2005)). It is well established that for the goods being perfect substitutes and the firms being quantity setters, the leader is better off than the follower because the cross-effect is positive. In case of Bertrand competition the opposite holds.

In most of the literature on industrial organization the strategy space is exogeneously given whereas the implications of endogeneously determined strategy spaces is rarely discussed. Based on a horizontally differentiated duopoly model by Dixit (1979), Singh and Vives (1984) considered a model in which the strategy space (price or quantity) is endogeneously chosen. The firms are allowed to offer two types of binding contracts to the consumers, i.e. a price or quantity contract in the first stage and in the second stage, the market stage, the firms compete simultaneously contingent on the type of contract. They showed that it is a dominant strategy for a firm to strategically set the quantity (price) if the goods are substitutes (complements). Boyer and Moreaux (1987) transferred the endogeneously determined strategy spaces into the leader-follower model and compared consumer, producer and total surplus with the values for the Nash equilibrium of the corresponding simultaneous move game. Using a very restrictive demand structure $^{1}$ they showed that it is always more profitable to be a quantity (price) setter if the goods are substitutes (complements). Concerning total and consumer surplus they proved that price competition is dominant for all degrees of product differentiation. Furthermore, they derive a unique ranking of the leader's and follower's prices, quantities and profits depending on the type of competition and the products being complements or substitutes. ${ }^{2}$

The purpose of this paper is to provide these comparisons for a more general demand structure introduced by Dixit (1979) with different cross-effects and reservation prices for the goods. It is shown that some of Boyer and Moreaux's results are still valid in this more general framework, while others are not.

\footnotetext{
${ }^{1}$ In this setting the degree of product differentiation and reservation prices are perfectly correlated.

${ }^{2}$ For further details see Boyer and Moreaux (1987) Propositions 1 and 2.
} 


\section{The sequential-move game with endogeneous strat- egy space}

Consider an economy with a monopolistic sector and two firms, each one producing a horizontal differentiated good, and a competitive numeraire sector as introduced by Dixit (1979). Following Singh and Vives (1984) and Boyer and Moreaux (1987) each firm selects whether to behave as a price or quantity setter. Additionally, it is assumed that the firms move sequentially: Firm 1 is the leader and firm 2 the follower. In particular, this is a Stackelberg setting with endogenous strategy spaces. Contingent on the strategy space decision, the price or quantity is chosen optimally. The game structure and some notations are summarized in figure 5.1. For instance, if firm 1 sets a price and firm 2 sets a quantity then $\pi_{i}^{p q}$ $\left(q_{i}^{p q}, p_{i}^{p q}\right)$ denotes firm $i$ 's profit (quantity, price).

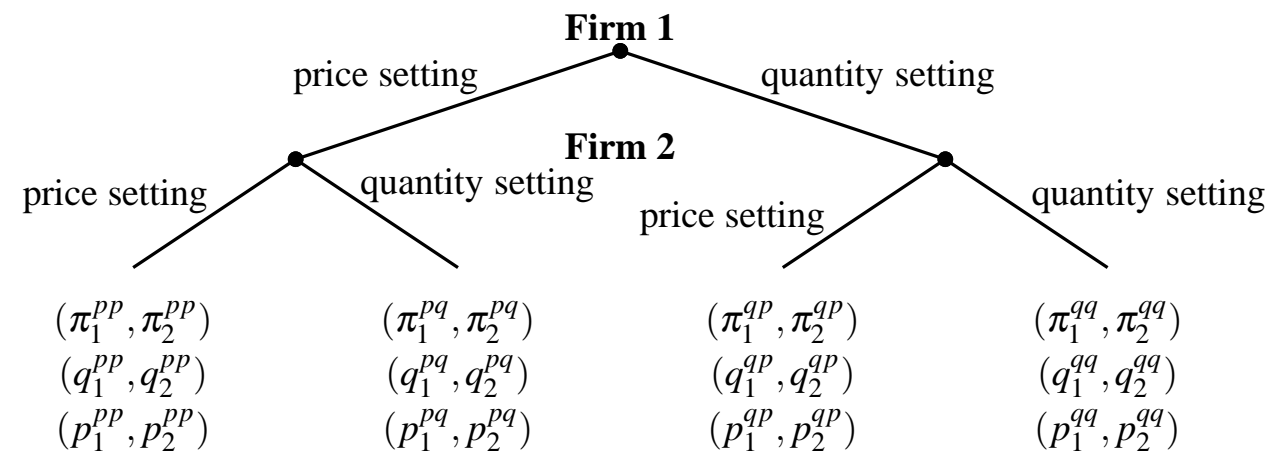

Figure 1: The game structure and notation.

The utility function of the representative consumer is assumed to be quadratic and strictly concave and given by

$$
u\left(q_{1}, q_{2}\right)=\alpha_{1} q_{1}+\alpha_{2} q_{2}-\frac{\beta_{1} q_{1}^{2}+2 \gamma q_{1} q_{2}+\beta_{2} q_{2}^{2}}{2}-\sum_{i=1}^{2} p_{i} q_{i}
$$

with $\alpha_{i}, \beta_{i} \in \mathbb{R}_{+}, i=1,2, \beta_{1} \beta_{2}-\gamma^{2}>0$ (concavity condition) and $\alpha_{i} \beta_{j}-\alpha_{j} \gamma>0$ (positive market size). In order to ensure these conditions, attention is restricted to $\gamma \in\left(-\sqrt{\beta_{1} \beta_{2}}, \min \left\{\sqrt{\beta_{1} \beta_{2}}, \frac{\alpha_{1} \beta_{2}}{\alpha_{2}}, \frac{\alpha_{2} \beta_{1}}{\alpha_{1}}\right\}\right)=: \Gamma$.

Moreover, utility maximization of the representative consumer gives rise to a linear demand structure

$$
\tilde{q}_{i}\left(p_{i}, p_{j}\right)=a_{i}-b_{i} p_{i}+c p_{j}, \quad i, j=1,2, \quad i \neq j,
$$


with $a_{i}=\frac{\alpha_{i} \beta_{j}-\alpha_{j} \gamma}{\beta_{1} \beta_{2}-\gamma^{2}}>0, b_{i}=\frac{\beta_{j}}{\beta_{1} \beta_{2}-\gamma^{2}}>0$ and $c=\frac{\gamma}{\beta_{1} \beta_{2}-\gamma^{2}}$. The corresponding inverse demand system is

$$
\tilde{p}_{i}\left(q_{i}, q_{j}\right)=\alpha_{i}-\beta_{i} q_{i}-\gamma q_{j}, \quad i, j=1,2, \quad i \neq j .
$$

The degree of product differentiation is determined by $\gamma$ : the goods are complements, independent or substitutes according to whether $\gamma \lesseqgtr 0$. Demand for good $i$ is downward sloping in its own price and increasing (decreasing) in the competitor's price if the goods are substitutes (complements). The goods are perfect substitutes whenever $\alpha_{1}=\alpha_{2}$ and $\beta_{1}=\beta_{2}=\gamma$. Moreover, for $\alpha_{1}=\alpha_{2}=\frac{1}{1+\alpha}$ $\beta_{1}=\beta_{2}=\frac{1}{1-\alpha^{2}}$ and $\gamma=-\frac{\alpha}{1-\alpha^{2}}$, the demand structure is equal to the one studied by Boyer and Moreaux (1987).

Firms have constant marginal costs, $c_{1}, c_{2} \geq 0$. W.o.l.g. it is assumed that prices are net of marginal costs. ${ }^{3}$ Then, profit of firm $i$ are given by $\pi_{i}=p_{i} q_{i}$. Following Singh and Vives (1984), the firms can offer two different types of contracts with the consumers: a price and a quantity contract. If a firm chooses to offer the price contract, then the firm will have to supply that amount which the consumers demand at a predetermined price independently of the competitor's action. If a firm chooses to offer the quantity contract, then the firm has to supply a predetermined quantity independently of the competitor's action. Moreover, still following Singh and Vives (1984), it is assumed that the costs associated with changing the type of contract are extremely high such that firms make the decision about the type contract once and then stick to it. Hence, each firm faces a problem two-stages: first the firms decide about the type of contract offered to the consumers, and afterwards they compete contingent on their chosen types of contract.

In case of pure quantity competition equation (2) is used for the profit maximization of the firms, whereas equation (1) is used in case of pure price competition. If one firm $i$ acts as price setter and firm $j$ chooses a quantity, a third system is introduced, which simply can be derived by using equations (1) and (2):

$$
\begin{aligned}
& \hat{q}_{i}\left(p_{i}, q_{j}\right)=\frac{\alpha_{i}-\gamma q_{j}-p_{i}}{\beta_{i}}, \\
& \hat{p}_{j}\left(p_{i}, q_{j}\right)=\frac{a_{j}+c p_{i}-q_{j}}{b_{j}}, \quad i, j=1,2, \quad i \neq j .
\end{aligned}
$$

W.o.l.g. firm 1 is the Stackelberg leader and before deciding about its strategy space and (based on this) about its optimal contract offered to the consumers, he

\footnotetext{
${ }^{3}$ Since $c_{i} \geq 0$ one may replace $\alpha_{i}$ and $a_{i}$ by $\alpha_{i}-c_{i}$ and $a_{i}-b_{i} m_{i}+c m_{j}, i, j=1,2, i \neq j$, respectively.
} 
anticipates all possible reactions of the follower. In particular, the leader determines the follower's best responses (optimal quantity or optimal price) for both scenarios: Price or quantity setting of the leader. For instance, if the leader sets a price and the follower reacts by setting a quantity, then the follower's best response is

$$
q_{2}^{*}\left(p_{1}\right):=\arg \max _{q_{2} \geq 0}=q_{2} p_{2}\left(p_{1}, q_{2}\right)=\arg \max _{q_{2} \geq 0} q_{2} \frac{a_{2}+c p_{1}-q_{2}}{b_{2}} .
$$

Since $\pi_{2}^{p q}\left(p_{1}, q_{2}\right)=q_{2} \frac{a_{2}+c p_{1}-q_{2}}{b_{2}}$ is strictly concave in $q_{2}$, the first order condition, $\frac{\partial \pi_{2}^{p q}\left(p_{1}, q_{2}\right)}{\partial q_{2}}=0$, implies $q_{2}^{*}\left(p_{1}\right)=\frac{a_{2}+c p_{1}}{2}$. The remaining best responses of the follower are derived analogously:

\begin{tabular}{|c|r|r|}
\hline Follower & Price Setting & Quantity Setting \\
\hline Price Setting & $\begin{array}{r}p_{2}^{*}\left(p_{1}\right)=\frac{a_{2}+c p_{1}}{2 b_{2}} \\
=\frac{\alpha_{2} \beta_{1}-\alpha_{1} \gamma+\gamma p_{1}}{2 \beta_{2}}\end{array}$ & $\begin{array}{r}q_{2}^{*}\left(p_{1}\right)=\frac{a_{2}+c p_{1}}{2} \\
=\frac{\alpha_{2} \beta_{1}-\gamma \alpha_{1}+\gamma p_{1}}{2\left(\beta_{1} \beta_{2}-\gamma^{2}\right)}\end{array}$ \\
\hline Quantity Setting & $p_{2}^{*}\left(q_{1}\right)=\frac{\alpha_{2}-\gamma q_{1}}{2}$ & $q_{2}^{*}\left(q_{1}\right)=\frac{\alpha_{2}-\gamma q_{1}}{2 \beta_{2}}$ \\
\hline
\end{tabular}

Table 1: The follower's best responses.

Next, the leader uses these best responses (of the follower) to derive his own optimal strategy. In particular, this means that the leader determines the optimal values of the strategic variable (price or quantity) for all four cases. For instance, if the follower sets a quantity and the leader would act as price setter, then the profit maximizing price is satisfied

$$
p_{1}^{p q}=\underset{p_{1} \geq 0}{\arg \max _{1}} \pi_{1}^{p q}\left(p_{1}, q_{2}^{*}\left(p_{1}\right)\right)=\underset{p_{1} \geq 0}{\arg \max _{1}} p_{1} \tilde{q}_{1}\left(p_{1}, q_{2}^{*}\left(p_{1}\right)\right) .
$$

As for the follower, since $\pi_{1}^{p q}\left(p_{1}, q_{2}^{*}\left(p_{1}\right)\right)=p_{1}\left(a_{1}-b_{1} p_{1}+c q_{2}^{*}\left(p_{1}\right)\right)$ is strictly concave in $p_{1}$, the first order condition, $\frac{\partial \pi_{1}^{p q}\left(p_{1}, q_{2}^{*}\left(p_{1}\right)\right)}{\partial p_{1}}=0$, yields $p_{1}^{p q}=\frac{2 a_{1} b_{2}+c a_{2}}{2\left(2 b_{1} b_{1}-c^{2}\right)}$. the remaining optimal values of the leader are derived analogously. 


\begin{tabular}{|c|c|c|}
\hline Follower & Price Setting & Quantity Setting \\
\hline Price Setting & $p_{1}^{p p}=\frac{2 a_{1} b_{2}+c a_{2}}{2\left(2 b_{1} b_{2}-c^{2}\right)}=\frac{2 \beta_{1} \beta_{2} \alpha_{1}-\beta_{1} \alpha_{2} \gamma-\alpha_{1} \gamma^{2}}{2\left(2 \beta_{1} \beta_{2}-\gamma^{2}\right)}=p_{1}^{p q}$ \\
\hline Quantity Setting & $q_{1}^{q p}=\frac{2 \beta_{2} \alpha_{1}-\alpha_{2} \gamma}{2\left(2 \beta_{1} \beta_{2}-\gamma^{2}\right)}=q_{1}^{q q}$ \\
\hline
\end{tabular}

Table 2: The leaders optimal values.

Table 2 shows that the value of the optimal strategic variable is independent of the followers strategy space. This leads to the following result:

Proposition 2.1. Independently of the follower's decision, the optimal prices, quantities and profits of both firms are predetermined by the leader's decision:

(i) If the leader chooses to set a price, then

$$
p_{i}^{p p}=p_{i}^{p q}, \quad q_{i}^{p p}=q_{i}^{p q} \quad \text { and } \quad \pi_{i}^{p p}=\pi_{i}^{p q}, \quad i=1,2 .
$$

(ii) If the leader chooses to set a quantity, then

$$
p_{i}^{q q}=p_{i}^{q p}, \quad q_{i}^{q q}=q_{i}^{q p} \quad \text { and } \quad \pi_{i}^{q q}=\pi_{i}^{q p}, \quad i=1,2 .
$$

Proof: Solving the leader's first order conditions imply equation (4). Plugging these optimal values into the follower's best replies imply equation (5).

After the leader has made the initial decision in the first stage, the follower acts as a monopolist on the remaining market. Therefore, price and quantity setting of the follower yield the same outcome. Hence, the maximal profit of the follower is predetermined by the action of the market leader, who is able to anticipate the follower's best replies to the different types of contracts.

Proposition 2.1 implies that only two distinct values of the strategic variables are possible depending on the leader's choice: One is for the case in which firm 1 sets a price, denoted by the upper index $p$, and a second case in which firm 1 
chooses a quantity, denoted by upper index $q$. This means:

$$
\begin{aligned}
\left(\pi_{1}^{p}, \pi_{2}^{p}\right) & :=\left(\pi_{1}^{p p}, \pi_{2}^{p p}\right)=\left(\pi_{1}^{p q}, \pi_{2}^{p q}\right) \\
\left(q_{1}^{p}, q_{2}^{p}\right) & :=\left(q_{1}^{p p}, q_{2}^{p p}\right)=\left(q_{1}^{p q}, q_{2}^{p q}\right) \\
\left(p_{1}^{p}, p_{2}^{p}\right) & :=\left(p_{1}^{p p}, p_{2}^{p p}\right)=\left(p_{1}^{p q}, p_{2}^{p q}\right) \\
\left(\pi_{1}^{q}, \pi_{2}^{q}\right) & :=\left(\pi_{1}^{q q}, \pi_{2}^{q q}\right)=\left(\pi_{1}^{q p}, \pi_{2}^{q p}\right) \\
\left(q_{1}^{q}, q_{2}^{q}\right) & :=\left(q_{1}^{q q}, q_{2}^{q q}\right)=\left(q_{1}^{q p}, q_{2}^{q p}\right) \\
\left(p_{1}^{q}, p_{2}^{q}\right) & :=\left(p_{1}^{q q}, p_{2}^{q q}\right)=\left(p_{1}^{q p}, p_{2}^{q p}\right) .
\end{aligned}
$$

Comparing these two scenarios leads to the following result:

Proposition 2.2. For the goods being substitutes (complements) the leader's price, quantity and corresponding profit are higher under quantity (price) setting than under price (quantity) setting. Under quantity (price) leadership also the follower's profit and price are higher than that under price (quantity) leadership of firm 1, while its quantity is always lower under quantity leadership than under price leadership.

The leader's decision on the type of contract solely depends on the degree of product differentiation, i.e. whether the goods are complements or substitutes. The comparison of all variables is summarized in Figure 2.2.

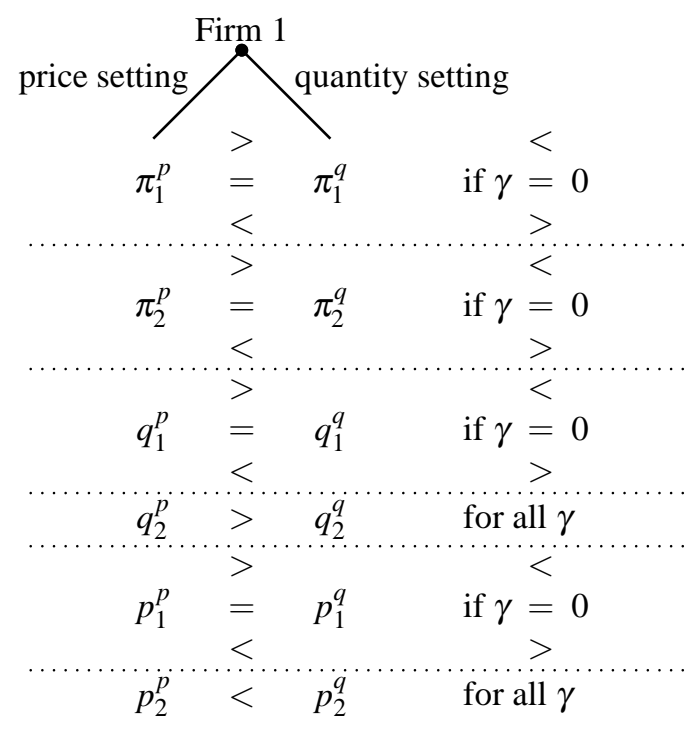

Figure 2: Comparison of case 1 and 2

A direct implication of Proposition 2.2 is that if goods are substitutes (complements), then producers' surplus, i.e. the sum of firms' profits, is higher in the 
quantity (price) leadership than in price (quantity) leadership. Only if goods are independent, i.e. $\gamma=0$ (both firms are monopolists), the firms are indifferent to the strategic variables.

As mentioned above, if $\alpha_{1}=\alpha_{2}=\frac{1}{1+\alpha}, \beta_{1}=\beta_{2}=\frac{1}{1-\alpha^{2}}$ and $\gamma=-\frac{\alpha}{1-\alpha^{2}}$ then the current demand structure is equal to that studied in Boyer and Moreaux (1987). Hence, their demand structure is a special case of that by Dixit (1979) which is applied in the current work. Moreover, it is easy to see that for parameterization the reservation prices and the degree of product differentiation are perfectly correlated. Therefore, the unique rankings of the leader's and follower's prices, quantities and profits purely depend on the type of competition (price or quantity leadership) and the degree of product differentiation (complements or substitutes).

Result 1 (Boyer and Moreaux (1987)).

(i) Price leader:

$$
p_{1}^{p}>p_{2}^{p} \text { and } q_{1}^{p}<q_{2}^{p} \text { for all } \gamma \text { and } \pi_{1}^{p}\left\{\begin{array}{c}
> \\
<
\end{array}\right\} \pi_{2}^{p} \text { if and only if } \gamma\left\{\begin{array}{c}
< \\
>
\end{array}\right\} 0 \text {. }
$$

(ii) Quantity leader:

$$
p_{1}^{q}<p_{2}^{q} \text { and } q_{1}^{q}>q_{2}^{q} \text { for all } \gamma \text { and } \pi_{1}^{q}\left\{\begin{array}{l}
> \\
<
\end{array}\right\} \pi_{2}^{q} \text { if an only if } \gamma\left\{\begin{array}{l}
> \\
<
\end{array}\right\} 0 \text {. }
$$

The following examples show that these rankings do not hold for the more general demand structure used here. ${ }^{4}$

Example 1 (quantity leader). Set $\alpha_{1}=\beta_{1}=1$.

1. Prices: For $\beta_{2}=\frac{1}{4}$ and $\alpha_{2}=\frac{1}{2}$ it is $p_{1}^{q}>p_{2}^{q} \forall \gamma$.

2. Quantities: For $\beta_{2}=1$ and $\alpha_{2}=2$ it is $q_{1}^{q}<q_{2}^{q} \forall \gamma$.

3. Profits: (a) For $\beta_{2}=1$ and $\alpha_{2}=2$ it is $\pi_{1}^{q}<\pi_{2}^{q} \forall \gamma$.

(b) For $\beta_{2}=1$ and $\alpha_{2}=\frac{1}{2}$ it is $\pi_{1}^{q}>\pi_{2}^{q} \forall \gamma$.

Example 2 (price leader). Set $\alpha_{1}=\beta_{1}=1$.

1. Prices: For $\beta_{2}=4$ and $\alpha_{2}=2$ it is $p_{1}^{p}<p_{2}^{p} \forall \gamma$.

\footnotetext{
${ }^{4}$ There are a lot of possible calibrations. The particular parameter calibrations in these examples are chosen because the rankings hold for all degrees of product differentiation $\gamma$.
} 
2. Quantities: For $\beta_{2}=1$ and $\alpha_{2}=\frac{1}{2}$ it is $q_{1}^{p}>q_{2}^{p} \forall \gamma$.

3. Profits: (a) For $\beta_{2}=1 / 4$ and $\alpha_{2}=1 / 2$ it is $\pi_{1}^{p}>\pi_{2}^{p} \forall \gamma$.

(b) For $\beta_{2}=4$ and $\alpha_{2}=2$ it is $\pi_{1}^{p}<\pi_{2}^{p} \forall \gamma$.

The examples suggest that the ordering of the market sizes, i.e. the reservation prices of the horizontal differentiated good, is crucial for the profits of the two duopolists. In particular, in Example 2.3 (a) firm 1 can be interpreted as an established firm whereas firm 2 represents a start-up facing a lower reservation price due to the fact that consumers are not willing to pay as much as they do for the horizontal differentiated good offered by the established firm $1\left(\alpha_{2}=0.5<\alpha_{1}=1\right)$. Furthermore, the consumers' sensitivity to small price changes can be measured by the absolute value of the slope of the (direct) demand function: The steeper the slope, the more sensitive is the consumers' demand to small changes in the price. In 2.3 (a) this means that the consumers' demand for the 'start-up product' is more sensitive than their demand for the 'established product'. In this case the profit of firm 2 is lower than firm 1's profit. Vice versa in 2.3 (b): the start-up evolves and firm 2's market size increases due to more acceptance and marketability of its product. This comes along with a lower sensitivity of the consumers' demand behaviour related to firm 2 . Therefore, the former start-up firm 2 now makes more profit than the etablished firm 1.

The last result in this section compares the consumers' and total surplus under price and quantity leadership: ${ }^{5}$

Proposition 2.3. Total and consumers' surplus are always higher under price leadership than under quantity leadership, regardless of the goods being complements or substitutes. Only if the goods are independent quantity competition is as good as price competition in terms of total and consumer surplus.

This result confirms and generalizes the result of Boyer and Moreaux (1987) who showed that consumers' and total surplus are higher under price leadership than under quantity leadership independently of the goods being substitutes or complements. Singh and Vives (1984) show, that that the analogue true in their simultaneous move game: price competition dominates quantity competition in terms of consumers' and total surplus.

\footnotetext{
${ }^{5}$ Here total surplus is equivalent to welfare, i.e. the sum of profits and consumer surplus.
} 


\section{Comparison between sequential and simultaneous move game}

The previous section has shown that some but not all of the results of Boyer and Moreaux (1987) can be confirmed in the current model. In particular, the previous section compared the two possible Stackelberg equilibria, given by $p_{i}^{p}, q_{i}^{p}$ and $\pi_{i}^{p}$ in case of price leadership and $p_{i}^{q}, q_{i}^{q}$ and $\pi_{i}^{q}$ under quantity leadership. Still following Boyer and Moreaux (1987) this section provides a comparison of these Stackelberg equilibria and the Nash equilibria of the underlying simultaneous move games as introduced by Singh and Vives (1984). Therefore, the analysis again must distinguish between four different cases: both set quantities, both set prices and two cases in which firm $i$ sets the price and firm $j$ sets the quantity. Following Singh and Vives (1984) it is:

\begin{tabular}{|c|c|}
\hline Type of Competition & Optimal Values \\
\hline Price Competition & $p_{i}^{B B}=\frac{2 a_{i} b_{j}+c a_{j}}{4 b_{i} b_{j}-c^{2}}=\frac{2 \alpha_{i} \beta_{i} \beta_{j}-\alpha_{i} \gamma^{2}-\beta_{i} \alpha_{j} \gamma}{4 \beta_{i} \beta_{j}-\gamma^{2}}$, \\
(Bertrand Competition) & $q_{i}^{B B}=b_{i} p_{i}^{B}=\frac{\beta_{j}\left(2 \alpha_{i} \beta_{i} \beta_{j}-\beta_{i} \alpha_{j} \gamma-\alpha_{i} \gamma^{2}\right)}{4 \beta_{i}^{2} \beta_{j}^{2}-5 \beta_{i} \beta_{j} \gamma^{2}+\gamma^{4}}$, \\
\hline$\pi_{i}^{B B}=$ & $p_{i}^{B B} q_{i}^{B B}$ for $i, j=1,2, \quad i \neq j$. \\
\hline Quantity Competition & $p_{i}^{C C}=\frac{\beta_{i}\left(2 \alpha_{i} \beta_{j}-\alpha_{j} \gamma\right)}{4 \beta_{i} \beta_{j}-\gamma^{2}}$, \\
(Cournot Competition) & $q_{i}^{C C}=\frac{2 \alpha_{i} \beta_{j}-\alpha_{j} \gamma}{4 \beta_{i} \beta_{j}-\gamma^{2}}$, \\
\hline$\pi_{i}^{C C}=$ & $p_{i}^{C C} q_{i}^{C C}$ for $i, j=1,2, \quad i \neq j$. \\
\hline Price vs. Quantity Setter & Firm $i: p_{i}^{B C}=\frac{2 a_{i} b_{j}+a_{j} c}{4 b_{i} b_{j}-3 c^{2}}=\frac{2 \alpha_{i} \beta_{i} \beta_{j}-\alpha_{i} \gamma^{2}-\alpha_{j} \beta_{i} \gamma}{4 \beta_{i} \beta_{j}-3 \gamma^{2}}$, \\
(firm $i$ price, firm $j$ quantity) & $q_{i}^{B C}=\frac{b_{i} b_{j}-c^{2}}{b_{j}} p_{i}^{B C}=\frac{2 \alpha_{i} \beta_{i} \beta_{j}-\alpha_{j} \beta_{i} \gamma-\alpha_{i} \gamma^{2}}{\beta_{i}\left(4 \beta_{i} \beta_{j}-3 \gamma^{2}\right)}$, \\
& $\pi_{i}^{B C}=p_{i}^{C B} q_{i}^{C B}$ \\
& Firm $j: p_{j}^{B C}=\frac{2 a_{j} b_{i}+a_{i} c-\frac{a_{j} c^{2}}{b_{j}}}{4 b_{i} b_{j}-3 c^{2}}=\frac{\left(2 \alpha_{j} \beta_{i}-\alpha_{i} \gamma\right)\left(\beta_{i} \beta_{j}-\gamma^{2}\right)}{\beta_{i}\left(4 \beta_{i} \beta_{j}-3 \gamma^{2}\right)}$ \\
& $q_{j}^{B C}=b_{j} p_{j}^{B C}=\frac{2 \alpha_{j} \beta_{i}-\alpha_{i} \gamma}{4 \beta_{i} \beta_{j}-3 \gamma^{2}}$, \\
$\pi_{j}^{B C}$ & $=p_{j}^{B C} q_{j}^{B C}$ for $i, j=1,2, \quad i \neq j$. \\
\hline
\end{tabular}

Table 3: The optimal values of the simultaneous move game. 
The comparison of these equilibrium values of the simultaneous move game and the equilibrium values of the Stackelberg game confirms Boyer and Moreaux's result that the equilibrium prices and quantities of the Stackelberg game are bounded from above and below by the equilibrium prices and quantities of the simultaneous move game, respectively. In particular,

\section{Proposition 3.1.}

1. Under price leadership in the Stackelberg game the following holds

$$
\begin{gathered}
p_{1}^{B B}<p_{1}^{p}<p_{1}^{B C} \text { and } q_{1}^{B C}<q_{1}^{p}<q_{1}^{B B} \quad \forall \gamma, \\
p_{2}^{B B} \stackrel{(>)}{<} p_{2}^{p} \stackrel{(>)}{<} p_{2}^{B C} \text { and } q_{2}^{B B} \stackrel{(>)}{<} q_{2}^{p} \stackrel{(>)}{<} q_{2}^{B C} \text { for } \quad \gamma^{(<)}>0, \\
\pi_{1}^{B C}<\pi_{1}^{B B}<\pi_{1}^{p} \quad \text { and } \pi_{2}^{B B} \stackrel{(>)}{<} \pi_{2}^{p} \stackrel{(>)}{<} \pi_{2}^{B C} \text { for } \quad \gamma \stackrel{(<)}{>} 0 .
\end{gathered}
$$

2. Quantity leadership implies

$$
\begin{gathered}
q_{1}^{C C}<q_{1}^{q}<q_{1}^{C B} \text { and } p_{1}^{C B}<p_{1}^{q}<p_{1}^{C C}, \\
p_{2}^{C C} \stackrel{(<)}{>} p_{2}^{q} \stackrel{(<)}{>} p_{2}^{C B} \text { and } q_{2}^{C C} \stackrel{(<)}{>} q_{2}^{q} \stackrel{(<)}{>} q_{2}^{C B} \text { for } \quad \gamma^{(<)}>0, \\
\pi_{1}^{C B}<\pi_{1}^{C C}<\pi_{1}^{q} \forall \gamma \text { and } \pi_{2}^{C B} \stackrel{(>)}{<} \pi_{2}^{q} \stackrel{(>)}{<} \pi_{2}^{C C} \text { for } \quad{ }^{(<)}>0 .
\end{gathered}
$$

This result partially confirms Boyer and Moreaux (1987) under weaker conditions in which no correlations between cross-effects and market size exist (compare Proposition V in Boyer and Moreaux (1987)). Moreover, since the leader's profit is larger than the profit in the simultaneous move game (compare equation (8) and (11)), the advantage of being the leader (compared to the situation in the simultaneous move game) is also reflected the leader's profit. A further implication of Proposition 3.1 is that if the goods are substitutes (complements), then the producers' surplus is higher in the Stackelberg game under price (quantity) leadership than in the Bertrand (Cournot) equilibrium of the simultaneous move game. For an illustration of Proposition 3.1 consider the following example.

Example 3. Consider $\alpha_{1}=\alpha_{2}=4, \beta_{1}=\beta_{2}=2$. This implies that $\frac{\gamma^{2}}{\beta_{1} \beta_{2}}=\frac{\gamma^{2}}{4}>$ 0 measures the degree of product differentiation. For this parametrization the equations (6), (7) and (8) yield the following figures 3,4 and $5 .^{6}$ 

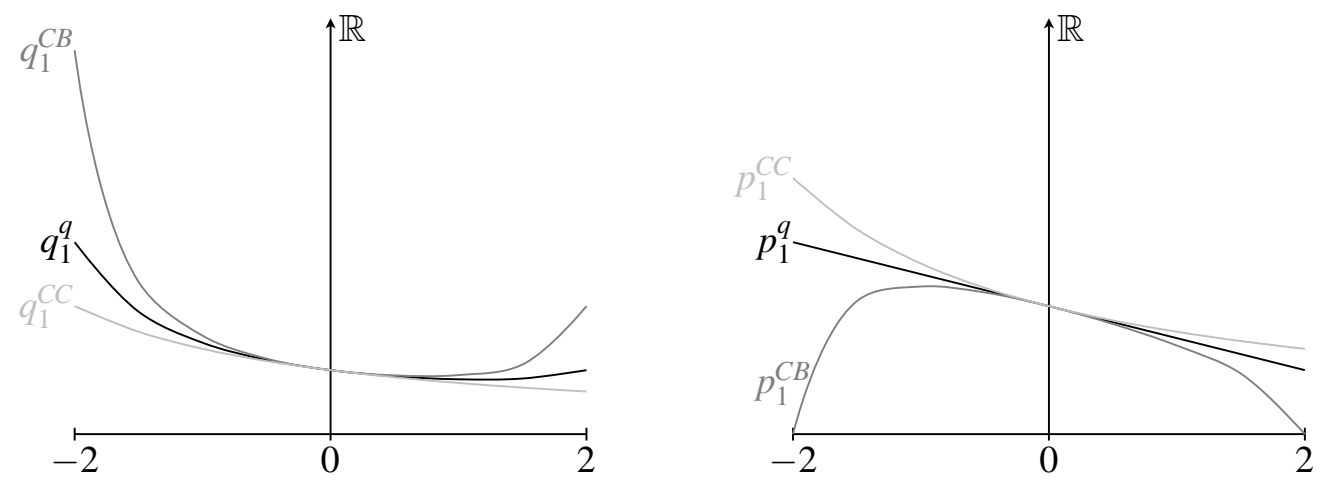

Figure 3: Quantity and price of firm 1.


Figure 4: Quantity and price of firm 2.
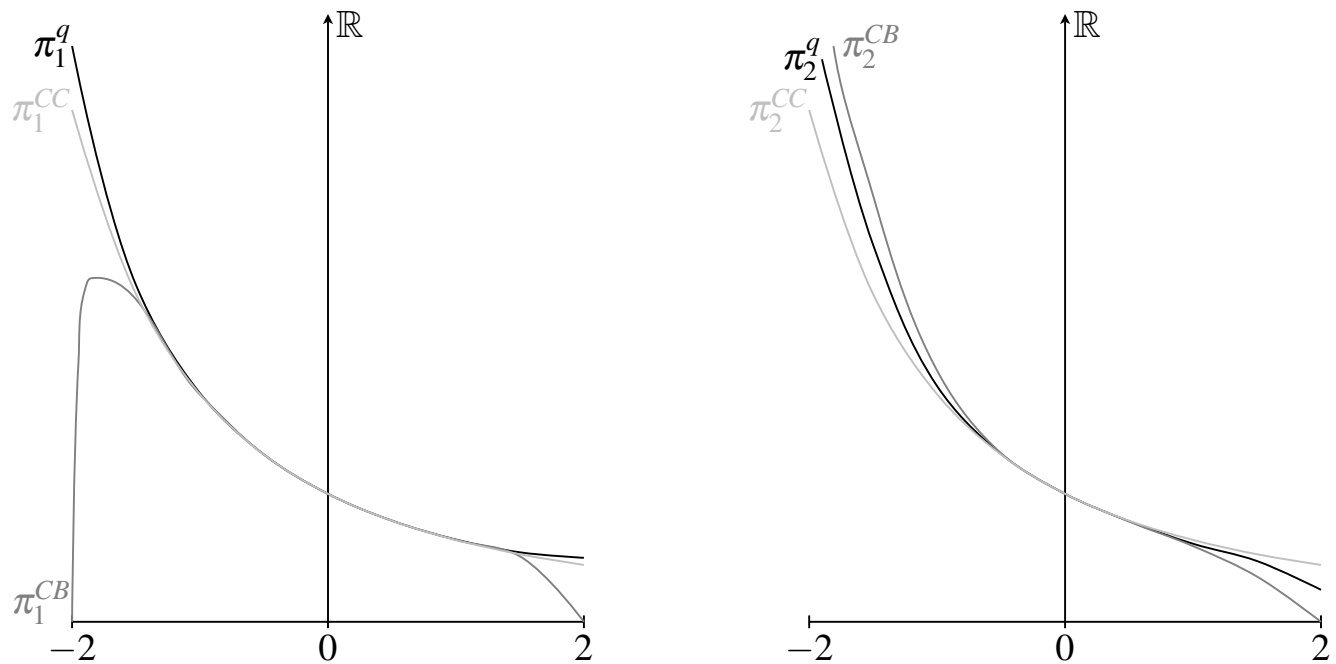

Figure 5: Profits of firm 1 and 2. 
Finally, the last result compares total and consumers' surplus in the Stackelberg game with those in the simultaneous move games. Loosely speaking it is shown that consumers' and total surplus are increasing in the number of pricesetting firms.

Proposition 3.2. Total and consumer surplus are always highest in the Bertrand equilibrium and lowest in the Cournot equilibrium. In between, Stackelberg equilibrium under price leadership dominates quantity leadership. ${ }^{7}$

This generalizes the results of Boyer and Moreaux (1987), i.e. total and consumer surplus increase in the number of price-setting firms. Moreover, both, Proposition 3.2 and the result of Vives (1985), imply that simultaneous Bertrand competition is optimal in terms of welfare and consumer surplus.

\section{Concluding Remarks}

In this paper the model by Boyer and Moreaux (1987) is generalized by using a less restrictive utility function introduced by Dixit (1979). The implications of this more general utility function are twofold: First, the demands for the two goods as a function of prices do not coincide and second, the cross-effects are different. In contrast to Boyer and Moreaux (1987) it is shown that the leader's price (quantity) in the price Stackelberg model is not necessarily higher (lower) than the follower's one. In contrast to Boyer and Moreaux (1987), the ordering of price, quantity and profit of the different simultaneous move (Cournot, Bertrand, mixed) and leaderfollower games by Boyer and Moreaux (1987) are verified only for firm 1. For firm 2 these orderings depend on both: the market size and the nature of the goods (substitutes or complements). Last, it is shown that price setting of at least one firm is preferable in terms of welfare and consumers' surplus independently of the game structure (simultaneous or sequential). Or loosely speaking: consumers' and total surplus are increasing in the number of price-setting firms.

\footnotetext{
${ }^{6}$ Examples for (9), (10), and (11) are omitted.

${ }^{7}$ The rankings of the mixed Nash equilibria and the Stackelberg equilibria depend on the exact parameterization.
} 


\section{A Proofs and algebraic manipulations}

Proof of Proposition 2.2 Simple algebraic calculations lead to the following differences in prices, quantities and profits of case 1 and 2:

$$
\begin{aligned}
& \Delta_{\pi_{1}}:=\pi_{1}^{p}-\pi_{1}^{q}=\gamma^{3} \overbrace{\underbrace{\frac{\beta_{1} \alpha_{2}^{2} \gamma+\beta_{2} \alpha_{1}^{2} \gamma-2 \beta_{1} \beta_{1} \alpha_{1} \alpha_{2}}{8 \beta_{1} \beta_{2}\left(\beta_{1} \beta_{2}-\gamma^{2}\right)\left(2 \beta_{1} \beta_{2}-\gamma^{2}\right)}}_{>0}}^{<0}, \\
& \Delta_{\pi_{2}}:=\pi_{2}^{p}-\pi_{2}^{q}=\gamma^{5} \underbrace{\overbrace{\beta_{1} \alpha_{2}^{2} \gamma+\beta_{2} \alpha_{1}^{2} \gamma-2 \beta_{1} \beta_{1} \alpha_{1} \alpha_{2}}^{<0}}_{>0}, \\
& \Delta_{p_{1}}:=p_{1}^{p}-p_{1}^{q}=\gamma^{3} \underbrace{\frac{\overbrace{-\alpha_{2}}^{<0}}{4 \beta_{2}\left(2 \beta_{1} \beta_{2}-\gamma^{2}\right)}}_{>0}, \\
& \Delta_{p_{2}}:=p_{2}^{p}-p_{2}^{q}=\gamma^{2} \underbrace{\frac{\overbrace{\alpha_{1} \gamma-2 \alpha_{2} \beta_{1}}^{<0}}{4 \beta_{1}\left(2 \beta_{1} \beta_{2}-\gamma^{2}\right)}}_{>0}, \\
& \Delta_{q_{1}}:=q_{1}^{p}-q_{1}^{q}=\gamma^{3} \underbrace{\frac{\overbrace{\alpha_{1} \gamma-\alpha_{2} \beta_{1}}^{<0}}{\beta_{1}\left(\beta_{1} \beta_{2}-\gamma^{2}\right)\left(2 \beta_{1} \beta_{2}-\gamma^{2}\right)}}_{>0}, \\
& \Delta_{q_{2}}:=q_{2}^{p}-q_{2}^{q}=\gamma^{2} \underbrace{\overbrace{2 \beta_{1} \beta_{2} \alpha_{2}-\beta_{2} \alpha_{1} \gamma-\alpha_{2} \gamma^{2}}^{40}}_{>0} .
\end{aligned}
$$

By using this it follows

$$
\Delta_{\pi_{1}}\left\{\begin{array} { l l } 
{ < 0 } & { \text { for } \gamma > 0 } \\
{ = 0 } & { \text { for } \gamma = 0 } \\
{ > 0 } & { \text { for } \gamma < 0 , }
\end{array} \quad \Delta _ { \pi _ { 2 } } \left\{\begin{array}{ll}
<0 & \text { for } \gamma>0 \\
=0 & \text { for } \gamma=0 \\
>0 & \text { for } \quad \gamma<0
\end{array}\right.\right.
$$




$$
\begin{aligned}
& \Delta_{p_{1}}\left\{\begin{array}{ll}
<0 & \text { for } \gamma>0 \\
=0 & \text { for } \gamma=0 \\
>0 & \text { for } \gamma<0,
\end{array} \quad \Delta_{p_{2}}<0 \text { for all } \gamma,\right. \\
& \Delta_{q_{1}}\left\{\begin{array}{ll}
<0 & \text { for } \gamma>0 \\
=0 & \text { for } \gamma=0 \\
>0 & \text { for } \gamma<0,
\end{array} \quad \Delta_{q_{2}}>0 \text { for all } \gamma\right. \text {. }
\end{aligned}
$$

Proof of Proposition 2.3 Consumers' surplus: The idea of the proof is to show that the difference in consumers' surplus $d(\gamma)=u$ ("price leadership") $u$ ("quantity leadership"), as a function of $\gamma$ has a global minimum at $\gamma=0$. Since $d(0)=0$ this implies the claim.

For $l \in\{p, q\}$ define

$$
u^{l}(\gamma):=\left.u\left(q_{1}^{l}, q_{2}^{l}\right)\right|_{p_{1}=p_{1}^{l}, p_{2}=p_{2}^{l}} .
$$

For $l=p(l=q)$, then $u^{p}(\gamma)\left(u^{p}(\gamma)\right)$ describes the consumer's utility in equilibrium as a function of $\gamma$ if firm 1 acts as price (quantity) setter. Now, by using the equilibium values it follows

$$
\begin{aligned}
u^{p}(\gamma)= & \frac{1}{32 \beta_{1}\left(2 \beta_{1} \beta_{2}-\gamma^{2}\right)^{2}\left(\beta_{1} \beta_{2}-\gamma^{2}\right)}\left[-3 \alpha_{1}^{2} \gamma^{6}+2 \alpha_{1} \alpha_{2} \beta_{1} \gamma^{5}+\left(16 \alpha_{1}^{2} \beta_{1} \beta_{2}+5 \alpha_{2}^{2} \beta_{1}^{2}\right) \gamma^{4}\right. \\
& \left.-4 \alpha_{1} \alpha_{2} \beta_{1}^{2} \beta_{2} \gamma^{3}-\left(28 \alpha_{1}^{2} \beta_{1}^{2} \beta_{2}^{2}+20 \alpha_{2}^{2} \beta_{1}^{3} \beta_{2}\right) \gamma^{2}+16\left(\alpha_{1}^{2} \beta_{1}^{3} \beta_{2}^{3}+\alpha_{2}^{2} \beta_{1}^{4} \beta_{2}^{2}\right)\right]
\end{aligned}
$$

and

$$
\begin{aligned}
u^{q}(\gamma)= & \frac{1}{32} \frac{1}{\left(2 \beta_{1} \beta_{2}-\gamma^{2}\right)^{2} \beta_{2}}\left[5 \alpha_{2}^{2} \gamma^{4}+4 \alpha_{1} \alpha_{2} \beta_{2} \gamma^{3}-\left(20 \alpha_{2}^{2} \beta_{1} \beta_{2}+12 \alpha_{1}^{2} \beta_{2}^{2}\right) \gamma^{2}\right. \\
& \left.+16\left(\alpha_{1}^{2} \beta_{1} \beta_{2}^{3}+\alpha_{2}^{2} \beta_{1}^{2} \beta_{2}^{2}\right)\right] .
\end{aligned}
$$

The 'utility-difference-function' is defined by

$$
\begin{aligned}
d(\gamma):= & u^{p}(\gamma)-u^{q}(\gamma) \\
= & \frac{\gamma^{2}}{32} \frac{1}{\beta_{1} \beta_{2}\left(2 \beta_{1} \beta_{2}-\gamma^{2}\right)^{2}\left(\beta_{1} \beta_{2}-\gamma^{2}\right)}\left[\left(5 \alpha_{2}^{2} \beta_{1}-3 \alpha_{1}^{2} \beta_{2}\right) \gamma^{4}+6 \alpha_{1} \alpha_{2} \beta_{1} \beta_{2} \gamma^{3}\right. \\
& \left.+\left(4 \alpha_{1}^{2} \beta_{1} \beta_{2}^{2}-20 \alpha_{2}^{2} \beta_{1}^{2} \beta_{2}\right) \gamma^{2}-8 \alpha_{1} \alpha_{2} \beta_{1}^{2} \beta_{2}^{2} \gamma+16 \alpha_{2}^{2} \beta_{1}^{3} \beta_{2}^{2}\right]
\end{aligned}
$$


The first and second derivatives are equal to

$$
\begin{aligned}
d^{\prime}(\gamma)= & \frac{\gamma}{16} \frac{1}{\left(\beta_{1} \beta_{2}-\gamma^{2}\right)^{2}\left(2 \beta_{1} \beta_{2}-\gamma^{2}\right)^{3}}\left[-3 \alpha_{1} \alpha_{2} \gamma^{7}+11\left(\beta_{1} \alpha_{1}^{2}-5 \beta_{1} \alpha_{2}^{2}\right) \gamma^{6}\right. \\
& -9 \beta_{1} \beta_{2} \alpha_{1} \alpha_{2} \gamma^{5}+\left(38 \beta_{1}^{2} \beta_{2} \alpha_{2}^{2}-26 \beta_{1} \beta_{2}^{2} \alpha_{1}^{2}\right) \gamma^{4}+34 \beta_{1}^{2} \beta_{2}^{2} \alpha_{1} \alpha_{2} \gamma^{3} \\
& \left.+\left(16 \beta_{1}^{2} \beta_{2}^{3} \alpha_{1}^{2}-64 \beta_{1}^{3} \beta_{2}^{2} \alpha_{2}^{2}\right) \gamma^{2}-24 \beta_{1}^{3} \beta_{2}^{3} \alpha_{1} \alpha_{2} \gamma+32 \beta_{1}^{4} \beta_{2}^{3} \alpha_{2}^{2}\right]
\end{aligned}
$$

and

$$
\begin{aligned}
d^{\prime \prime}(\gamma)= & \frac{1}{16\left(\beta_{1} \beta_{2}-\gamma^{2}\right)^{3}\left(2 \beta_{1} \beta_{2}-\gamma^{2}\right)^{4}}\left[6 \alpha_{1} \alpha_{2} \gamma^{11}+\left(15 \alpha_{2}^{2} \beta_{1}-33 \alpha_{1}^{2} \beta_{2}\right) \gamma^{10}+66 \alpha_{1} \alpha_{2} \beta_{1} \beta_{1} \gamma^{9}\right. \\
& +\left(53 \alpha_{1}^{2} \beta_{1} \beta_{2}^{2}-155 \alpha_{2}^{2} \beta_{1}^{2} \beta_{2}\right) \gamma^{8}-216 \alpha_{1} \alpha_{2} \beta_{1}^{2} \beta_{2}^{2} \gamma^{7}+\left(68 \alpha_{1}^{2} \beta_{1}^{2} \beta_{2}^{3}+340 \alpha_{2}^{2} \beta_{1}^{3} \beta_{2}^{2}\right) \gamma^{6} \\
& +152 \alpha_{1} \alpha_{2} \beta_{1}^{3} \beta_{2}^{3} \gamma^{5}-\left(228 \alpha_{2}^{2} \beta_{1}^{4} \beta_{2}^{3}-180 \alpha_{1}^{2} \beta_{1}^{3} \beta_{2}^{4}\right) \gamma^{4}+80 \alpha_{1} \alpha_{2} \beta_{1}^{4} \beta_{2}^{4} \gamma^{3} \\
& \left.+\left(96 \alpha_{1}^{2} \beta_{1}^{4} \beta_{2}^{5}-32 \alpha_{2}^{2} \beta_{1}^{5} \beta_{2}^{4}\right) \gamma^{2}-96 \alpha_{1} \alpha_{2} \beta_{1}^{5} \beta_{2}^{5} \gamma+64 \alpha_{2}^{2} \beta_{1}^{6} \beta_{2}^{5}\right] .
\end{aligned}
$$

Since $d^{\prime}(0)=0$ and $d^{\prime \prime}(0)=\frac{\alpha_{2}^{2}}{4 \beta_{1} \beta_{2}^{2}}>0$ it follows that $d(0)=0$ is a local minimum of $d(\gamma)$, i.e. $\exists \varepsilon>0$ s.t. $u^{p}(\gamma)>u^{q}(\gamma) \forall \gamma \in(-\varepsilon, \varepsilon)$.

Moreover, since $\alpha_{1}, \alpha_{2}, \beta_{1}, \beta_{2} \in \mathbb{R}$ and $\gamma \in \Gamma$, Lemma A.1 (which is stated directly below this proof) implies

$$
d(\gamma)=0 \Leftrightarrow u^{p}(\gamma)=u^{q}(\gamma) \Leftrightarrow \gamma=0,
$$

i.e. $\gamma=0$ is the only root of $d(\gamma)$ in $\Gamma$. This implies that $\gamma=0$ is a global minimum of $d(\gamma)$ which proves the claim.

Total surplus: Analog to the proof for consumers' surplus.

The proof of Proposition 2.3 makes use of the following Lemma:

Lemma A.1. $d(\gamma)>0$ for all $\gamma \in \Gamma \backslash\{0\}$.

Proof of Lemma A.1 Define

$$
\begin{aligned}
h(\gamma):= & \underbrace{\left(5 \alpha_{2}^{2} \beta_{1}-3 \alpha_{1}^{2} \beta_{2}\right)}_{=: A_{1}} \gamma^{4}+\underbrace{6 \alpha_{1} \alpha_{2} \beta_{1} \beta_{2}}_{=: A_{2}} \gamma^{3}+\underbrace{\left(4 \alpha_{1}^{2} \beta_{1} \beta_{2}^{2}-20 \alpha_{2}^{2} \beta_{1}^{2} \beta_{2}\right)}_{=: A_{3}} \gamma^{2} \\
& \underbrace{-8 \alpha_{1} \alpha_{2} \beta_{1}^{2} \beta_{2}^{2}}_{=: A_{4}} \gamma+\underbrace{16 \alpha_{2}^{2} \beta_{1}^{3} \beta_{2}^{2}}_{=: A_{5}} .
\end{aligned}
$$


Equation (12) implies

$$
d(\gamma)=0 \text { for } \gamma \neq 0 \Leftrightarrow h(\gamma)=0 .
$$

1. $\frac{\alpha_{1}}{\alpha_{2}} \leq \sqrt{\frac{\beta_{1}}{\beta_{2}}}$ : In this case $\Gamma=\left(-\sqrt{\beta_{1} \beta_{2}}, \frac{\alpha_{1}}{\alpha_{2}} \beta_{2}\right)$.

A) $0<\gamma \in \Gamma$ : Since

$$
h^{\prime}(\gamma)=\underbrace{4 A_{1}}_{+} \gamma^{3}+\underbrace{3 A_{2}}_{+} \gamma^{2}+\underbrace{2 A_{3}}_{-} \gamma+\underbrace{A_{4}}_{-}
$$

Descarte's rule (see e.g. David J. Grabiner: Descartes' Rule of Signs. Another Construction. In: American Mathematical Monthly, Jg. 106 (1999), S. 854-855), implies that $h^{\prime}(\gamma)$ has exactly one positive root. Since

$$
h^{\prime}(0)=-8 \alpha_{1} \alpha_{2}\left(\beta_{1} \beta_{2}\right)^{2}<0
$$

and

$$
h^{\prime}\left(\frac{\alpha_{1}}{\alpha_{2}} \beta_{2}\right)=\frac{2 \alpha_{1} \beta_{2}^{2}}{\alpha_{2}^{3}}\left[23 \alpha_{1}^{2} \alpha_{2}^{2}-24 \alpha_{2}^{4} \beta_{1}^{2}-6 \alpha_{1}^{4} \beta_{2}^{2}\right]<0
$$

this implies $h^{\prime}(\gamma)<0$ for all $\gamma \in\left(0, \frac{\alpha_{1}}{\alpha_{2}} \beta_{2}\right)$. Hence,

$$
h(\gamma)>h\left(\frac{\alpha_{1}}{\alpha_{2}} \beta_{2}\right)=\underbrace{-\frac{\beta_{2}^{2}}{\alpha_{2}^{4}}}_{-} \underbrace{\left(\alpha_{1}^{2} \beta_{2}-\alpha_{2}^{2} \beta_{1}\right)}_{-} \underbrace{\left(3 \alpha_{1}^{4} \beta_{2}^{2}-12 \alpha_{1}^{2} \alpha_{2}^{2} \beta_{1} \beta_{2}+16 \alpha_{2}^{4} \beta_{1}^{2}\right)}_{+}>0,
$$

where

$$
3 \alpha_{1}^{4} \beta_{2}^{2}-12 \alpha_{1}^{2} \alpha_{2}^{2} \beta_{1} \beta_{2}+16 \alpha_{2}^{4} \beta_{1}^{2}=\left(\alpha_{1}+x_{1}\right)\left(\alpha_{1}-x_{1}\right)\left(\alpha_{1}+x_{2}\right)\left(\alpha_{1}-x_{2}\right)>0
$$

with $x_{1}:=\sqrt{\frac{6+2 I \sqrt{3}}{3} \frac{\beta_{1}}{\beta_{2}}} \alpha_{2}$ and $x_{2}:=\sqrt{\frac{6-2 I \sqrt{3}}{3} \frac{\beta_{1}}{\beta_{2}}} \alpha_{2}$, holds because

$$
\lim _{\alpha_{1} \rightarrow 0, \alpha_{1}>0} 3 \alpha_{1}^{4} \beta_{2}^{2}-12 \alpha_{1}^{2} \alpha_{2}^{2} \beta_{1} \beta_{2}+16 \alpha_{2}^{4} \beta_{1}^{2}=16 \alpha_{2}^{4} \beta_{1}^{2}>0 .
$$

B) $0>\gamma \in \Gamma:$ Since

$$
h^{\prime}(-\gamma)=\underbrace{-4 A_{1}}_{+} \gamma^{3}+\underbrace{3 A_{2}}_{+} \gamma^{2}+\underbrace{-2 A_{3}}_{-} \gamma+\underbrace{A_{4}}_{-}
$$

Descarte's rule implies that $h^{\prime}(\gamma)$ has exactly one negative root $\bar{\gamma}$. Since

$$
h^{\prime}(0)<0<h^{\prime}\left(-\sqrt{\beta_{1} \beta_{2}}\right)=2 \beta_{1} \beta_{2}\left[2 \sqrt{\beta_{1} \beta_{2}}\left(\alpha_{1}^{2} \beta_{2}+5 \alpha_{2}^{2} \beta_{1}\right)+5 \alpha_{1} \alpha_{2} \beta_{1} \beta_{2}\right]
$$


this implies $\bar{\gamma} \in\left(-\sqrt{\beta_{1} \beta_{2}}, 0\right)$. In combination with

$$
H\left(-\sqrt{\beta_{1} \beta_{2}}\right)=\left(\beta_{1} \beta_{2}\right)^{2}\left[2 \sqrt{\beta_{1} \beta_{2}} \alpha_{1} \alpha_{2}+\alpha_{1}^{2} \beta_{2}+\alpha_{2}^{2} \beta_{1}\right]>0 \text { and } h(0)>0
$$

this implies $h(\gamma)>0$ for all $\gamma \in\left(-\sqrt{\beta_{1} \beta_{2}}, 0\right)$.

2. $\sqrt{\frac{\beta_{1}}{\beta_{2}}}<\frac{\alpha_{1}}{\alpha_{2}} \leq \sqrt{\frac{5}{3} \frac{\beta_{1}}{\beta_{2}}}$ : In this case $\Gamma=\left(-\sqrt{\beta_{1} \beta_{2}}, \frac{\alpha_{2}}{\alpha_{1}} \beta_{1}\right)$. The rest of the proof is analogue to 1 .

3. $\sqrt{\frac{5}{3} \frac{\beta_{1}}{\beta_{2}}}<\frac{\alpha_{1}}{\alpha_{2}} \leq \sqrt{5 \frac{\beta_{1}}{\beta_{2}}}$ : In this case $\Gamma=\left(-\sqrt{\beta_{1} \beta_{2}}, \frac{\alpha_{2}}{\alpha_{1}} \beta_{1}\right)$.

A) $0<\gamma \in \Gamma$ : Since

$$
h^{\prime}(\gamma)=\underbrace{4 A_{1}}_{-} \gamma^{3}+\underbrace{3 A_{2}}_{+} \gamma^{2}+\underbrace{2 A_{3}}_{-} \gamma+\underbrace{A_{4}}_{-}
$$

Descarte's rule implies that $h^{\prime}(\gamma)$ has either 2 or no positive roots. If $h^{\prime}$ has no positive root, then $h^{\prime}(\gamma)<0$ and, hence, $h(\gamma)>h\left(\frac{\alpha_{2}}{\alpha_{1}} \beta_{1}\right)>0$. If $h^{\prime}$ has 2 positive roots, then there exist $\gamma_{1}<\gamma_{2}$ such that

$$
h^{\prime}(\gamma) \begin{cases}\geq 0 & \text { for } \gamma \in\left[\gamma_{1}, \gamma_{2}\right] \\ <0 & \text { else. }\end{cases}
$$

Therefore, there must be $\gamma_{3} \in\left(\gamma_{1}, \gamma_{2}\right)$ such that $h^{\prime \prime \prime}\left(\gamma_{3}\right)=0$ and $h^{i v}\left(\gamma_{3}\right)<0$. The solution of $h^{\prime \prime \prime}(\gamma)=0$ is

$$
\bar{\gamma}_{1 / 2}=\frac{9 \alpha_{1} \alpha_{2} \beta_{1} \beta_{2} \pm \sqrt{72 \alpha_{1}^{4} \beta_{1} \beta_{2}^{3}-399\left(\alpha_{1} \alpha_{2} \beta_{1} \beta_{2}\right)^{2}+600 \alpha_{2}^{4} \beta_{1}^{3} \beta_{2}}}{6\left(3 \alpha_{1}^{2} \beta_{2}-5 \alpha_{2}^{2} \beta_{1}\right)}>0 .
$$

Since

$$
\begin{aligned}
& h^{i v}\left(\frac{9 \alpha_{1} \alpha_{2} \beta_{1} \beta_{2}+\sqrt{72 \alpha_{1}^{4} \beta_{1} \beta_{2}^{3}-399\left(\alpha_{1} \alpha_{2} \beta_{1} \beta_{2}\right)^{2}+600 \alpha_{2}^{4} \beta_{1}^{3} \beta_{2}}}{6\left(3 \alpha_{1}^{2} \beta_{2}-5 \alpha_{2}^{2} \beta_{1}\right)}\right) \\
= & -4 \sqrt{3} \sqrt{24 \alpha_{1}^{4} \beta_{1} \beta_{2}^{3}-133\left(\alpha_{1} \alpha_{2} \beta_{1} \beta_{2}\right)^{2}+200 \alpha_{2}^{4} \beta_{1}^{3} \beta_{2}}<0
\end{aligned}
$$

and

$$
\begin{aligned}
& h^{i v}\left(\frac{9 \alpha_{1} \alpha_{2} \beta_{1} \beta_{2}+\sqrt{72 \alpha_{1}^{4} \beta_{1} \beta_{2}^{3}-399\left(\alpha_{1} \alpha_{2} \beta_{1} \beta_{2}\right)^{2}+600 \alpha_{2}^{4} \beta_{1}^{3} \beta_{2}}}{6\left(3 \alpha_{1}^{2} \beta_{2}-5 \alpha_{2}^{2} \beta_{1}\right)}\right) \\
= & 4 \sqrt{3} \sqrt{24 \alpha_{1}^{4} \beta_{1} \beta_{2}^{3}-133\left(\alpha_{1} \alpha_{2} \beta_{1} \beta_{2}\right)^{2}+200 \alpha_{2}^{4} \beta_{1}^{3} \beta_{2}}>0
\end{aligned}
$$


we get $\gamma_{3}=\frac{9 \alpha_{1} \alpha_{2} \beta_{1} \beta_{2}+\sqrt{72 \alpha_{1}^{4} \beta_{1} \beta_{2}^{3}-399\left(\alpha_{1} \alpha_{2} \beta_{1} \beta_{2}\right)^{2}+600 \alpha_{2}^{4} \beta_{1}^{3} \beta_{2}}}{6\left(3 \alpha_{1}^{2} \beta_{2}-5 \alpha_{2}^{2} \beta_{1}\right)}$. Now,

$$
\gamma_{3}-\frac{\alpha_{2}}{\alpha_{1}} \beta_{1}=\frac{9 \alpha_{1}^{2} \alpha_{2} \beta_{1} \beta_{2}-30 \alpha_{2}^{3} \beta_{1}^{2}-\sqrt{3} \alpha_{1} \sqrt{24 \alpha_{1}^{4} \beta_{1} \beta_{2}^{3}-133\left(\alpha_{1} \alpha_{2} \beta_{1} \beta_{2}\right)^{2}+200 \alpha_{2}^{4} \beta_{1}^{3} \beta_{2}}}{6\left(3 \alpha_{1}^{2} \beta_{2}-5 \alpha_{2}^{2} \beta_{1}\right)}>0
$$

implies $\gamma_{3} \notin \Gamma$. This implies $\gamma_{1}, \gamma_{2} \notin \Gamma$ and, hence, $h^{\prime}(\gamma)<0$ for all $0<\gamma \in \Gamma$. The rest of the proof is analogue to the 1.A).

B) $0>\gamma \in \Gamma:$ Since

$$
h(-\gamma)=\underbrace{A_{1}}_{-} \gamma^{4}+\underbrace{-A_{2}}_{-} \gamma^{3}+\underbrace{A_{3}}_{-} \gamma^{2}+\underbrace{-A_{4}}_{+} \gamma+\underbrace{A_{5}}_{+}
$$

Descarte's rule implies that $h$ has exactly one negative root. In combination with

$$
0<H(0) \text { and } h\left(-\sqrt{\beta_{1} \beta_{2}}\right)=\beta_{1}^{2} \beta_{2}^{2}\left[14 \sqrt{\beta_{1} \beta_{2}} \alpha_{1} \alpha_{2}+\alpha_{1}^{2} \beta_{2}+\alpha_{2}^{2} \beta_{1}\right]>0
$$

this implies $h(\gamma)>0$ for all $\gamma \in\left(-\sqrt{\beta_{1} \beta_{2}}, 0\right)$.

4. $\sqrt{5 \frac{\beta_{1}}{\beta_{2}}}<\frac{\alpha_{1}}{\alpha_{2}}$ : In this case $\Gamma=\left(-\sqrt{\beta_{1} \beta_{2}}, \frac{\alpha_{2}}{\alpha_{1}} \beta_{1}\right)$.

A) $0<\gamma \in \Gamma$ : Since

$$
h^{\prime}(\gamma)=\underbrace{4 A_{1}}_{-} \gamma^{3}+\underbrace{3 A_{2}}_{+} \gamma^{2}+\underbrace{2 A_{3}}_{+} \gamma+\underbrace{A_{4}}_{-}
$$

Descarte's rule implies that $h^{\prime}(\gamma)$ has either 2 or no positive roots. The rest of the proof is analogue to part 3.A).

B) $0>\gamma \in \Gamma$ : Analogue to part 3.B).

The results stated in Proposition 2.2 follow immediately.

Proof of Proposition 3.1 First, consider the equations (6), (7), and (8). The profit of firm 1 for the case if firm 2 chooses also price competition is given by table 1 :

$$
\begin{aligned}
\tilde{\pi}_{1}^{p p}\left(p_{1}\right) & :=\pi_{1}^{p p}\left(p_{1}, R_{2}^{p p}\left(p_{1}\right)\right) \\
& =p_{1} \tilde{q}_{1}\left(p_{1}, R_{2}^{p p}\left(p_{1}\right)\right) \\
& =p_{1}\left(a_{1}-b_{1} p_{1}+c R_{2}^{p p}\left(p_{1}\right)\right)
\end{aligned}
$$


with $R_{2}^{p p}\left(p_{1}\right)=\frac{a_{2}+c p_{1}}{2 b_{2}}=\frac{\alpha_{2} \beta_{1}-\alpha_{1} \gamma+\gamma p_{1}}{2 \beta_{2}}$ being the best reply. The first order condition can be written to

$$
\begin{aligned}
\frac{\partial \tilde{\pi}_{1}^{p p}}{\partial p_{1}} & =\underbrace{}_{\begin{array}{c}
\text { Direct effect } \\
\frac{\partial \pi_{1}^{p p}}{\partial p_{1}}
\end{array}+\underbrace{\frac{\partial \pi_{1}}{\partial p_{2}} \frac{\partial R_{2}^{p p}}{\partial p_{1}}}_{\text {Strategic effect }}} \\
& =\frac{\partial \pi_{1}^{p p}}{\partial p_{1}}+\frac{\gamma}{\beta_{1} \beta_{2}-\gamma^{2}} \frac{\gamma}{2 \beta_{1}} p_{1} .
\end{aligned}
$$

The strategic effect is positive for all feasible $\gamma$. In the equilibrium of the simultaneous Bertrand game on the second stage it holds that $\frac{\partial \pi_{1}^{p p}}{\partial p_{1}}\left(p_{1}^{B B}\right)=0$ which implies that

$$
\frac{\partial \tilde{\pi}_{1}^{p p}}{\partial p_{1}}\left(p_{1}^{B B}\right)=\frac{\gamma^{2}}{2 \beta_{1}\left(\beta_{1} \beta_{2}-\gamma^{2}\right)} p_{1}^{B B}>0 \text { for all } \gamma .
$$

As the profit function is concave it follows directly

$$
p_{1}^{B B}<p_{1}^{p} .
$$

The profit of firm 1 for the case if firm 2 chooses quantity competition yields

$$
\begin{aligned}
\tilde{\pi}_{1}^{p q}\left(p_{1}\right): & =\pi_{1}^{p q}\left(p_{1}, R_{2}^{p q}\left(p_{1}\right)\right) \\
& =p_{1} \hat{q}_{1}\left(p_{1}, R_{2}^{p q}\left(q_{1}\right)\right) \\
& =p_{1} \frac{\alpha_{1}-\gamma R_{2}^{p q}\left(q_{1}\right)-p_{1}}{\beta_{1}}
\end{aligned}
$$

with $R_{2}^{p q}\left(p_{1}\right)=\frac{a_{2}+c p_{1}}{2}=\frac{\alpha_{2} \beta_{1}-\gamma \alpha_{1}+\gamma p_{1}}{2\left(\beta_{1} \beta_{2}-\gamma^{2}\right)}$. The first order condition can be written to

$$
\begin{aligned}
\frac{\partial \tilde{\pi}_{1}^{p q}}{\partial p_{1}} & =\underbrace{\frac{\partial \pi_{1}^{p q}}{\partial p_{1}}}_{\text {Direct effect }}+\underbrace{\frac{\partial \pi_{1}}{\partial q_{2}} \frac{\partial R_{2}^{p q}}{\partial p_{1}}}_{\text {Strategic effect }} \\
& =\frac{\partial \pi_{1}^{p q}}{\partial p_{1}}-\frac{\gamma p_{1}}{\beta_{1}} \frac{\gamma}{2\left(\beta_{1} \beta_{2}-\gamma^{2}\right)} .
\end{aligned}
$$

The strategic effect is negative for all feasible $\gamma$. In the equilibrium of the simultaneous move it holds that $\frac{\partial \pi_{1}^{p q}}{\partial p_{1}}\left(p_{1}^{B C}\right)=0$ which implies that

$$
\frac{\partial \tilde{\pi}_{1}^{p q}}{\partial p_{1}}\left(p_{1}^{B C}\right)=-\frac{\gamma^{2}}{2 \beta_{1}\left(\beta_{1} \beta_{2}-\gamma^{2}\right)} p_{1}^{B C}<0 \text { for all } \gamma \text {. }
$$


It follows directly by concavity of the profit function that

$$
p_{1}^{B C}>p_{1}^{p}
$$

holds. Together with equation (15) this implies inequality (6). The other inequalities follow directly by using simple algebraic manipulations which are omitted here.

It remains to prove the equations (9), (10), (11). The profit of firm 1 for the case if both firms select quantity competition is given by:

$$
\begin{aligned}
\tilde{\pi}_{1}^{q q}\left(q_{1}\right): & =\pi_{1}^{q q}\left(q_{1}, R_{2}^{q q}\left(q_{1}\right)\right) \\
& =q_{1} \tilde{p}_{1}\left(q_{1}, R_{2}^{q q}\left(q_{1}\right)\right)
\end{aligned}
$$

with $R_{2}^{q q}\left(q_{1}\right)=\frac{\alpha_{2}-\gamma q_{1}}{2 \beta_{2}}$. The first order condition can be written to

$$
\begin{aligned}
\frac{\partial \tilde{\pi}_{1}^{q q}}{\partial q_{1}} & =\underbrace{\frac{\partial \pi_{1}^{q q}}{\partial q_{1}}}_{\begin{array}{c}
\text { Direct effect } \\
\text { Strategic effect }
\end{array}}+\underbrace{\frac{\partial \pi_{1}}{\partial q_{2}} \frac{\partial R_{2}^{q q}}{\partial q_{1}}} \\
& =\frac{\partial \pi_{1}^{q q}}{\partial q_{1}}+\frac{\gamma^{2}}{2 \beta_{2}} q_{1} .
\end{aligned}
$$

The strategic effect is positive for all feasible $\gamma$. In the equilibrium of the simultaneous Cournot game it holds that $\frac{\partial \pi_{1}^{q q}}{\partial q_{1}}\left(q_{1}^{C C}\right)=0$ which implies that

$$
\frac{\partial \tilde{\pi}_{1}^{q q}}{\partial q_{1}}\left(q_{1}^{C C}\right)=\frac{\gamma^{2}}{2 \beta_{2}} q_{1}^{C C}>0 \text { for all } \gamma .
$$

Concavity of the profit function implies

$$
q_{1}^{C C}<q_{1}^{q} .
$$

The profit of firm 1 for the case if firm 2 chooses price competition yields

$$
\begin{aligned}
\tilde{\pi}_{1}^{q p}\left(q_{1}\right): & =\pi_{1}^{q p}\left(q_{1}, R_{2}^{q p}\left(q_{1}\right)\right) \\
& =q_{1} \hat{p}_{1}\left(q_{1}, R_{2}^{q p}\left(q_{1}\right)\right)
\end{aligned}
$$

with $R_{2}^{q p}\left(q_{1}\right)=\frac{\alpha_{2}-\gamma q_{1}}{2}$. The first order condition can be written to

$$
\begin{aligned}
\frac{\partial \tilde{\pi}_{1}^{q p}}{\partial q_{1}} & =\underbrace{\frac{\partial \pi_{1}^{q p}}{\partial q_{1}}}_{\begin{array}{c}
\text { Direct effect } \\
\text { Strategic effect }
\end{array}}+\underbrace{\frac{\partial \pi_{1}}{\partial p_{2}} \frac{\partial R_{2}^{q p}}{\partial q_{1}}} \\
& =\frac{\partial \pi_{1}^{q p}}{\partial q_{1}}-\frac{\gamma^{2}}{2 \beta_{2}} q_{1} .
\end{aligned}
$$


Obviously, the strategic effect is negative for all feasible $\gamma$. In the equilibrium of the simultaneous move it holds that $\frac{\partial \pi_{1}^{q p}}{\partial q_{1}}\left(p_{1}^{C B}\right)=0$ which implies that

$$
\frac{\partial \tilde{\pi}_{1}^{p q}}{\partial q_{1}}\left(q_{1}^{C B}\right)=-\frac{\gamma^{2}}{2 \beta_{2}} q_{1}^{C B}<0 \text { for all } \gamma
$$

It follows directly by concavity of the profit function that

$$
q_{1}^{C B}>q_{1}^{q} .
$$

Together with equation (17) this implies the second inequality of equation (6). The other inequalities follow analogoulsy.

The explicit algebraic expressions of figures 3, 4 and 5 in Example 3:

$$
\begin{gathered}
q_{1}^{q}=\frac{16-4 \gamma}{2\left(8-\gamma^{2}\right)}, \quad q_{1}^{C C}=\frac{16-4 \gamma}{16-\gamma^{2}}, \quad q_{1}^{C B}=\frac{16-4 \gamma}{16-3 \gamma^{2}} \\
p_{1}^{q}=\frac{4-\gamma}{2}, \quad p_{1}^{C C}=\frac{8}{4+\gamma}, \quad p_{1}^{C B}=\frac{2(4-\gamma)\left(4-\gamma^{2}\right)}{16-3 \gamma^{2}} \\
q_{2}^{q}=\frac{16-4 \gamma-\gamma^{2}}{2\left(8-\gamma^{2}\right)}, q_{2}^{C C}=\frac{16-4 \gamma}{16-\gamma^{2}}, q_{2}^{C B}=\frac{2\left(8-2 \gamma-\gamma^{2}\right)}{16-3 \gamma^{2}} \\
p_{2}^{q}=\frac{16-4 \gamma-\gamma^{2}}{8-\gamma^{2}}, \quad p_{2}^{C C}=\frac{8}{4+\gamma}, \quad p_{2}^{C B}=\frac{4\left(8-\gamma^{2}\right)-8 \gamma}{16-3 \gamma^{2}} \\
\pi_{1}^{q}=\frac{(4-\gamma)^{2}}{8-\gamma^{2}}, \quad \pi_{1}^{C C}=\frac{32}{(4+\gamma)^{2}}, \quad \pi_{1}^{C B}=\frac{8(4-\gamma)^{2}\left(4-\gamma^{2}\right)}{2\left(16-3 \gamma^{2}\right)^{2}} \\
\pi_{2}^{q}=\frac{(4-\gamma)^{2}}{8-\gamma^{2}}, \quad \pi_{2}^{C C}=\frac{32}{(4+\gamma)^{2}}, \quad \pi_{2}^{C B}=\frac{8\left(8-2 \gamma-\gamma^{2}\right)^{2}}{\left(16-3 \gamma^{2}\right)^{2}}
\end{gathered}
$$

Proof of Proposition 3.2 Analogue to the proof of Proposition 2.3. 


\section{References}

R. Amir and J. Y. Jin. Cournot and Bertrand equilibria compared: substitutability, complementary and concavity. International Journal of Industrial Organization, 19:303-317, 2001.

J. Betrand. Révue de la Théorie Mathématique de la Richesse Sociale et des Recherches sur les Principles Mathématiques de la Théorie des Richesses. Journal des Savants, pages 499-508, 1883.

M. Boyer and M. Moreaux. On Stackelberg equilibria with differentiated products: The critical role of the strategy space. The Journal of Industrial Economics, 36(2):217-230, 1987.

A. Cournot. Récherches sur les Principes Mathématiques de la Théorie des Richesses. Hachette, Paris, Paris, 1838.

G. K. Dastidar. On Stackelberg games in a homogeneous product market. European Economic Review, 48:549-562, 2004.

A. K. Dixit. A model of duopoly suggesting a theory of entry barriers. The Bell Journal of Economics, 10:20-32, 1979.

D.. Kreps and J. Scheinkman. Quantity precommitment and Bertrand competition yield Cournot outcomes. The Bell Journal of Economics, 14:326-337, 1983.

N. Singh and X. Vives. Price and quantity competition in a differentiated duopoly. The RAND Journal of Economics, 15(4):546-554, 1984.

H. Stackelberg. Marktform und Gleichgewicht. Springer, Vienna, 1934.

X. Vives. On the Efficency of Bertrand and Cournot Equilibria with Product Differentation. Journal of Economic Theory, 36:166-175, 1985.

X. Vives. Games with strategic complementarities: New applications to industrial organization. International Journal of Industrial Organization, 23:625-637, 2005. 